\title{
Nonequilibrium characterization of spinodal points using short time dynamics
}

\author{
Ernesto S. Loscar, ${ }^{1,2, a)}$ Ezequiel E. Ferrero, ${ }^{3}$ Tomás S. Grigera, ${ }^{1,2}$ and Sergio A. Cannas ${ }^{3}$ \\ ${ }^{1}$ Departamento de Física and Instituto de Investigaciones Fisicoquímicas Teóricas y Aplicadas \\ (INIFTA), Facultad de Ciencias Exactas, Universidad Nacional de La Plata, \\ c.c. 16, suc. 4, 1900 La Plata, Argentina \\ ${ }^{2}$ CCT La Plata, Consejo Nacional de Investigaciones Científicas y Técnicas (CONICET), 55 Street \#910, \\ 1900 La Plata, Argentina \\ ${ }^{3}$ Instituto de Física de la Facultad de Matemática, Astronomía y Física (IFFAMAF-CONICET), \\ Universidad Nacional de Córdoba, Ciudad Universitaria, 5000 Córdoba, Argentina
}

(Received 14 November 2008; accepted 12 June 2009; published online 14 July 2009)

\begin{abstract}
Although intuitively appealing, the concept of spinodal is rigorously defined only in systems with infinite range interactions (mean-field systems). In short-range systems, a pseudospinodal can be defined by extrapolation of metastable measurements, but the point itself is not reachable because it lies beyond the metastability limit. In this work we show that a sensible definition of spinodal points can be obtained through the short time dynamical behavior of the system deep inside the metastable phase by looking for a point where the system shows critical behavior. We show that spinodal points obtained by this method agree both with the thermodynamical spinodal point in mean-field systems and with the pseudospinodal point obtained by extrapolation of metaequilibrium behavior in short-range systems. With this definition, a practical determination can be achieved without regard for equilibration issues. (C) 2009 American Institute of Physics.
\end{abstract}

[DOI: $10.1063 / 1.3168404]$

\section{INTRODUCTION}

First order phase transitions are accompanied by hysteresis and metastability: Even though the thermodynamic transition happens at the value $\varphi_{t}$ of the control variable $\varphi$, when $\varphi$ is varied smoothly from $\varphi_{i}>\varphi_{t}$ to $\varphi_{f}<\varphi_{t}$, the system remains in the phase corresponding to thermodynamic equilibrium at $\varphi>\varphi_{t}$ (and conversely when changing $\varphi$ in the opposite sense). When the phase survives being carried out beyond its thermodynamic "homeland," it is called metastable. Metastable phases have a finite lifetime, but this time can be very long. Diamond at room temperature and pressure and glass-forming supercooled liquids are very well-known examples of long-lived metastable phases (so long lived, in fact, that for many purposes they can be considered as an equilibrium phase). In general, however, the metastable phase cannot exist for all $\varphi$, and it is not observed if $\varphi_{f}$ is less than some value $\varphi_{\mathrm{sp}}$. This is the idea behind the concept of spinodal point. However, to define the spinodal, some care is required.

At the mean-field level, the spinodal is well defined. Focusing on the ferromagnetic case to be specific, let us consider the extended free energy per particle ${ }^{1} f_{3}(T, m, h)$ with $h$ as the magnetic field and $m$ as the magnetization. $f_{3}$ depends on two conjugate variables because it is defined such that the probability of finding a value $M$ of the magnetic moment is $\propto \exp \left[-\beta N f_{3}(T, M / N, h)\right]$, where $N$ is the system size. In mean field and in the limit $N \rightarrow \infty, f_{3}$ has two minima as a function of $m$ for $T$ below some critical temperature $T_{c}$ and $h$

${ }^{a)}$ Electronic mail: yasser.loscar@gmail.com. within some range $-h_{\mathrm{sp}}<h<h_{\mathrm{sp}}{ }^{1,2}$ As a function of $h$, the (first order) transition occurs at $h=h_{c}=0$. When $h=0$, the two minima are symmetric, corresponding to the two brokensymmetry phases. When $h \neq 0$, the absolute minimum corresponds to the thermodynamic equilibrium (or stable) phase, while the secondary local minimum defines a phase which is dynamically stable but of higher free energy: The metastable phase. When it exists, the (mean-field) metastable phase has infinite lifetime. At $h=h_{\mathrm{sp}}$, the secondary minimum disappears (it becomes an inflection point) and the phase becomes unstable: A system prepared with a magnetization different from the (thermodynamic) equilibrium value evolves toward the equilibrium state. For $|h| \geq h_{\text {sp }}, f_{3}$ has only one minimum. Thus in mean field the spinodal, which is the point where the metastable phase becomes unstable (in the sense that a susceptibility becomes negative), is also the limit of metastability, i.e., the point up to which the metastable phase can be observed.

When the interactions have a finite range, matters are more complicated. $^{2,3}$ On one hand, the metastable phase ceases to be observable before it becomes unstable. ${ }^{4-6}$ This is because as the system moves away from the transition, the lifetime of the metastable phase decreases while its relaxation time increases. When they become of the same order, the phase is unobservable. This is the metastability limit, which is thus different from the spinodal. The metastability limit is also called kinetic spinodal, ${ }^{7}$ and the term thermodynamic spinodal is sometimes used for the spinodal as defined above (onset of an instability). On the other hand, the order parameter can fluctuate in space. Although $f_{3}$ can still be defined (and can be used to compute the true equilibrium 
properties), in the thermodynamic limit it has no convexity changes and only one minimum; therefore, it is useless to define a spinodal. Further, although the extensive $F_{3}$ has a double-well shape, ${ }^{8}$ the local maximum cannot be interpreted as a barrier to the growth of the stable phase because the global magnetization is not a good coordinate to describe this process: A supercritical nucleus of the stable phase (one whose growth is thermodynamically favored) ${ }^{9}$ can form without change in global magnetization. ${ }^{8}$ The eventual disappearance of the secondary minimum in $F_{3}$ is hence unrelated to the spinodal.

The spinodal is then beyond the metastability limit, and hence outside the realm of thermodynamics and of quasiequilibrium treatments. Due to these difficulties, it has been concluded that the spinodal only makes sense in mean-field ${ }^{10}$ or in finite size systems. ${ }^{3}$ However, signs of an instability are detectable in (meta)equilibrium measurements: The susceptibility and relaxation times of the metastable phase increase as one goes deeper into the metastable region, and if extrapolated with a power law, they seem to diverge at a point beyond the metastability limit called pseudospinodal. ${ }^{10,11}$

Here, rather than to abandon the idea of a spinodal in finite-range systems, we propose to define it through out-ofequilibrium properties. The idea is that the spinodal should be a point with infinite susceptibility and infinite relaxation time. In this sense it resembles the critical point of a second order phase transition. It is known ${ }^{12,13}$ that it is possible to detect critical points by studying the short time dynamic behavior of the order parameter and correlation functions. We propose to use the same method to identify a point deep into the metastable region which, at short times, behaves dynamically like a critical point. This pseudocritical regime (in the sense that it lasts only for a finite time) does not imply the existence of a thermodynamic singularity (see discussion in Sec. V). We show that in a mean-field model, where the spinodal is well defined and can be worked out analytically, the point identified with this technique is precisely the thermodynamic spinodal. Thus by defining the spinodal as the point where this pseudocritical dynamics takes place we provide a sensible generalization of the spinodal concept to finite-range systems. We apply the method to the twodimensional $q$-state Potts mode ${ }^{14}$ with $q>4$, where it gives a reasonable result, providing a bound for the metastability limit and locating the spinodal very near the pseudospinodal. The technique has the advantage that it does not need equilibrium data, which are an essential requirement in order to determine the pseudospinodal.

Our proposal was inspired by the finding of Schülke and Zheng ${ }^{15}$ that the STD applied to the Potts model defines two "pseudocritical points," which are closer together with the weaker (first order) transition and coincide for second order transitions. A similar situation was observed in models with out-of-equilibrium transitions. ${ }^{16,17}$

The paper is organized as follows. In Sec. II we briefly review the STD technique. In Sec. III we consider mean-field spin models and we show that the STD method accurately describes the thermodynamic spinodal in those models. In
Sec. IV we apply the method to the ferromagnetic $q$-state Potts model with nearest-neighbor interactions in two dimensions. Section V presents our conclusions.

\section{USING SHORT TIME DYNAMICS TO IDENTIFY CRITICAL POINTS}

The STD technique to identify critical points has been reviewed in Refs. 13 and 18. Briefly, it is based on the work of Janssen et al., ${ }^{12}$ who studied model A (a $\phi^{4}$ Hamiltonian with Langevin dynamics) in the out-of-equilibrium regime where correlation functions are still nontrivial functions of two times and the order parameter is still time dependent. For the present work, the relevant result is that the $n$th moment of the order parameter $m^{(n)}(t)=\left\langle[m(t)-\langle m(t)\rangle]^{n}\right\rangle$ obeys the scaling form ${ }^{12,18}$

$$
m^{(n)}\left(t, \tau, L, m_{0}\right)=b^{-n \beta / v} g_{n}\left(b^{-z} t, b^{1 / \nu} \tau, L / b, b^{\mu} m_{0}\right),
$$

where $t$ is the time, $\tau$ is the reduced temperature $\tau=(T$ $\left.-T_{c}\right) / T_{c}, L$ is the system size, $m_{0}$ is the initial value of the order parameter (assumed nonzero but small), and $b$ is a rescaling parameter. $\mu$ is a new universal exponent that describes the short time behavior, while $\beta, \nu$, and $z$ are the usual critical exponents. ${ }^{1}$

From Eq. (1), setting $b=t^{1 / z}$, for large values of $L$ and small values of $t^{1 / z} m_{0}$ one obtains

$$
m\left(t, \tau, m_{0}\right) \sim m_{0} t^{\theta} F\left(t^{1 / \nu z} \tau\right), \quad \theta=\frac{\mu-\beta \nu}{z},
$$

so that precisely at the critical point $\tau=0$, the order parameter obeys a power law $m(t) \sim t^{\theta}$. Similarly, setting $m_{0}=0$, one gets for the second moment at the critical point

$$
m^{(2)}(t) \sim t^{d / z-2 \beta / z \nu} .
$$

It is generally assumed (and in agreement with numerical results ${ }^{13,18}$ ) that when the initial condition is the ordered state $\left(m_{0}=1\right)$, a scaling similar to Eq. (1) holds

$$
m^{(n)}(t, \tau, L)=b^{-n \beta / \nu} g_{n}\left(b^{-z} t, b^{1 / \nu} \tau, L / b\right) .
$$

From this equation one obtains, for $m_{0}=1$ and large $L$,

$$
m(t)=t^{-\beta / v z} G\left(t^{1 / v z} \tau\right)
$$

and taking the derivative of $\log m$,

$$
\left.\frac{\partial \log m(t, \tau)}{\partial \tau}\right|_{\tau=0} \sim t^{1 / \nu \tau} .
$$

The critical point can then be obtained by performing Monte Carlo simulations at several temperatures and looking for the value of $T$ at which the power laws in time predicted by Eqs. (2), (3), and (5) hold. In addition, these equations together with Eq. (6) allow to determine the critical exponents. ${ }^{13}$

Here we apply the above method to look for a singular behavior in the metastable region of a first order phase transition. By tuning the appropriate control parameter (external field or temperature) we look for a value where the power laws (2), (3), and (5) hold at short time scales (for very long times, the approach to the proper equilibrium phase is seen). This value of the control parameter can be sensibly defined as a spinodal, as we show in the cases studied below. 


\section{SPINODAL POINTS IN SYSTEMS WITH LONG RANGE INTERACTIONS}

In this section we consider spin models where each spin interacts with every other spin. For those systems mean-field theory is exact and therefore provides a first check for the STD method. Let us first briefly review the analytical meanfield results on the spinodal points.

\section{A. Thermodynamic spinodals \\ 1. Curie-Weiss-Ising model}

We first consider the Curie-Weiss or fully connected version of the Ising model. In the presence of an external magnetic field $h$ the Hamiltonian is given by

$$
\mathcal{H}_{\mathrm{CWI}}=-\frac{J}{2 N} \sum_{i \neq j} s_{i} s_{j}-h \sum_{i=1}^{N} s_{i}
$$

where $N$ is the number of spins $\left(s_{i}= \pm 1\right), h$ is an external magnetic field, and $J>0$. The extended free energy per spin $f_{3}$ can be computed exactly because $\mathcal{H}_{\mathrm{CW}}$ is an explicit function of the total magnetization $M=\sum_{i} s_{i}$ and the partition function can be evaluated for fixed $M$. The result in the limit $N \rightarrow \infty$ is

$f_{3}(T, m, h)=\frac{1}{\beta}\left[\frac{1+m}{2} \ln \frac{1+m}{2}+\frac{1-m}{2} \ln \frac{1-m}{2}\right]-\frac{J}{2} m^{2}-h m$,

where $\beta=1 / T$ (we take Boltzmann's constant equal to 1 ) and $m=M / N$. The absolute minimum of $f_{3}$ with respect to $m$ defines the stable (equilibrium) solution $m(T, h)$. The model shows a second order transition at $h=h_{c}=0$ and critical temperature $T_{c}=J$. For $T<T_{c}$, there is a line of first order transitions at $h=0$, where $m(T, h)$ is singular. However, an analytic continuation $m_{+}(T, h)$ from positive to negative $h$ exists as long as $|h|$ is not too big [and conversely a continuation $m_{-}(T, h)$ from negative to positive $\left.h\right]$. These continuations are the metastable states and correspond to local minima of $f_{3}$. Thus the conditions

$$
\frac{\partial f_{3}(T, m, h)}{\partial m}=0, \quad \frac{\partial^{2} f_{3}(T, m, h)}{\partial m^{2}}>0,
$$

define the (meta)stable states. The secondary minimum (and thus the metastable solution) ceases to exist when $h<h_{\mathrm{sp}}^{(-)}=-h_{\mathrm{sp}}$, the spinodal field, given by

$$
\left.\frac{\partial^{2} f_{3}(T, m, h)}{\partial m^{2}}\right|_{h=h_{\mathrm{sp}}^{(-)}}=0 .
$$

Since $\partial^{2} f_{3} / \partial m^{2}=\chi_{T}^{-1}$, the susceptibility diverges at $h_{\mathrm{sp}}^{(-)}$, and it is straightforward to show that for $h-h_{\mathrm{sp}}^{(-)} \ll 1$,

$$
\chi_{T} \sim\left(h-h_{\mathrm{sp}}^{(-)}\right)^{-1 / 2} .
$$

In fact, this singularity can be treated like a usual critical point. For example, for fixed $T$ we have

$$
C_{h} \sim\left(h-h_{\mathrm{sp}}^{(-)}\right)^{-1 / 2},
$$

$$
\Delta m \sim\left(h-h_{\mathrm{sp}}^{(-)}\right)^{1 / 2},
$$

where $C_{h}$ is the specific heat and $\Delta m=m-m_{\mathrm{sp}}^{(-)}$with $m_{\mathrm{sp}}^{(-)}$ $=m\left(h_{\mathrm{sp}}^{(-)}\right)$(note that $\Delta m>0$ ). Fixing $h$, an expansion in 0 $<T_{\mathrm{sp}}-T \ll 1$ gives

$$
\begin{aligned}
& \Delta m \sim\left(T_{\mathrm{sp}}-T\right)^{1 / 2}, \\
& C_{h} \sim\left(T_{\mathrm{sp}}-T\right)^{-1 / 2}, \\
& \chi_{T} \sim\left(T_{\mathrm{sp}}-T\right)^{-1 / 2} .
\end{aligned}
$$

If we choose $\Delta m$ as order parameter, the singular behavior in the neighborhood of the spinodal point can be characterized exactly as in a true critical point. The set of critical exponents is

$$
\beta=1 / 2, \quad \alpha=1 / 2, \quad \gamma=1 / 2, \quad \delta=2,
$$

which satisfies the Rushbrooke and Widom scaling relations

$$
\begin{aligned}
& \alpha+2 \beta+\gamma=2, \\
& \gamma=\beta(\delta-1) .
\end{aligned}
$$

If we assume that $\eta=0$ like in mean-field critical points, from the Fisher scaling relation $\gamma=\nu(2-\eta)$, we have $\nu=1 / 4$. Finally, from the Josephson hyperscaling,

$$
\nu d=2-\alpha,
$$

we can guess a critical dimension $d_{c}=6$, a result confirmed by a renormalization group analysis. ${ }^{19}$

\section{Curie-Weiss-Potts model}

The Curie-Weiss-Potts model is defined by the Hamiltonian

$$
\mathcal{H}_{\mathrm{CWP}}=-\frac{J}{N} \sum_{i \neq j} \delta\left(\sigma_{i}, \sigma_{j}\right),
$$

where $J>0, \sigma_{i}=1,2, \ldots, q$, and $\delta\left(\sigma_{i}, \sigma_{j}\right)$ is the Kronecker delta. The extended free energy per spin $f_{3}$ for this model can also be computed exactly, ${ }^{14}$

$$
\begin{aligned}
f_{3}(m, T)= & -\frac{J(q-1)}{2 q} m^{2} \\
& +\frac{1}{\beta q}\left\{[1+(q-1) m] \ln \left[\frac{1+(q-1) m}{q}\right]\right. \\
& \left.+(q-1)(1-m) \ln \left[\frac{1-m}{q}\right]\right\} .
\end{aligned}
$$

The order parameter $m$ is defined as

$$
m=\frac{1}{q-1}\left(q\left\langle\max \left(m_{k}\right)\right\rangle-1\right),
$$

where $m_{k}=1 / N \Sigma_{i=1}^{N} \delta\left(\sigma_{i}, k\right), k=1 \cdots q$, and the maximum means choosing the value of $k$ that gives the highest value of $m_{k}$. The extended free energy (22) presents two local minima (and therefore a first order transition) for $q \geq 3$, where the transition happens at $T_{t}=J(q-2) \ln (q-1) / 2(q-1) .{ }^{20} \mathrm{~A}$ disordered metastable solution with $m=0$ (supercooled paramagnet) exists for $T_{\mathrm{sp}}^{(-)}<T<T_{t}$ (with $T_{\mathrm{sp}}^{(-)}=J / q$ ) as well as an 
ordered metastable solution with $m=m_{\mathrm{sp}} \neq 0$ for $T_{t}<T$ $<T_{\mathrm{sp}}^{(+)}$, where the spinodal temperatures are obtained from Eq. (10) with $h=0$. An analysis similar to the above shows that close to the upper spinodal $T_{\mathrm{sp}}^{(+)}$, the order parameter $\Delta m$, the specific heat, and the susceptibility show the same critical behavior [Eqs. (14)-(16)] with the same critical exponent (17). Of course, the same set of exponents is obtained from other mean-field approximations: The Landau $\phi^{4}$ model and, for the upper spinodal point, the Landau $\phi^{6}$ model. The values of these exponents are confirmed by means of renormalization group techniques. ${ }^{19,21}$

On the other hand, the behavior close to the lower spinodal temperature $T_{\mathrm{sp}}^{(-)}$is different. The susceptibility shows a power-law divergence with exponent $\gamma=1$, while the magnetization and the specific heat remain finite (in fact, they are identically zero in the disordered phase). The vanishing of the specific heat is peculiar to the approximation; the point is that in principle one should not expect to find the same critical exponent in both spinodals.

\section{B. Short time dynamics}

We now apply the STD procedure to the Curie-WeissIsing model in the neighborhood of the spinodal point using $\Delta m$ as an order parameter. We consider the process at fixed temperature with the magnetic field as the control variable. We choose the initial condition in the ordered state $(m=1)$ corresponding to $h \rightarrow \infty$. In this case, the procedure analog to a quench $T$ near $T_{c}$ for a thermal second order transition is to set $h$ to a value near $h=h_{\mathrm{sp}}^{(-)}$. To determine $h_{\mathrm{sp}}^{(-)}$, we assume a scaling like Eq. (1) with $\tau=\left(h-h_{\mathrm{sp}}^{(-)}\right) / h_{\mathrm{sp}}^{(-)}$.

In this test case, we can check the spinodal field and magnetization found with STD against the exact values obtained from Eqs. (9) and (10),

$$
\begin{aligned}
& m_{\mathrm{sp}}^{(-)}=\sqrt{1-\frac{T}{J}}, \\
& \beta h_{\mathrm{sp}}^{(-)}=\frac{1}{2} \ln \left[\frac{1+m_{\mathrm{sp}}^{(-)}}{1-m_{\mathrm{sp}}^{(-)}}\right]-\frac{m_{\mathrm{sp}}^{(-)} J}{T} .
\end{aligned}
$$

We have simulated the dynamics of this model using a standard Metropolis algorithm in a system of $N=1.6 \times 10^{6}$ spins. We started with all spins up and did $n \sim 10^{3}$ runs while recording $\Delta m$ and the second moment of the magnetization per spin $\Delta m^{(2)}$. Figure 1 shows the short time behavior of $\Delta m$ and $\Delta m^{(2)}$ for $T=\frac{4}{9} T_{c}$, where time is measured in Monte Carlo steps (MCSs) (one MCS is defined as a full cycle of $N$ spin update trials). From Eqs. (24) and (25) we have $\beta h_{\mathrm{sp}}^{(-)}$ $\cong-0.714627$ and $m_{\mathrm{sp}}^{(-)} \cong 0.745356$.

For both first and second moments [Eqs. (3) and (5)] a power law behavior $\Delta m \sim t^{-x}$ and $\Delta m^{(2)} \sim t^{y}$ at $h=h_{\mathrm{sp}}^{(-)}$is found with exponents $x=0.98 \pm 0.02$ and $y=1.03 \pm 0.02$, respectively. The power laws are rather short lived (lasting up to $t \sim 100 \mathrm{MCS})$ and both observables deviate afterward. However, as we will show below this is a finite size effect, and a true power law should be observed in the thermodynamic limit. In order to calculate the derivative of $\log \Delta m$ from simulations we have taken the magnetic field very close to the spinodal field, that is $h=h_{\mathrm{sp}} \pm \epsilon$ with $\epsilon=2 \times 10^{-4}$.
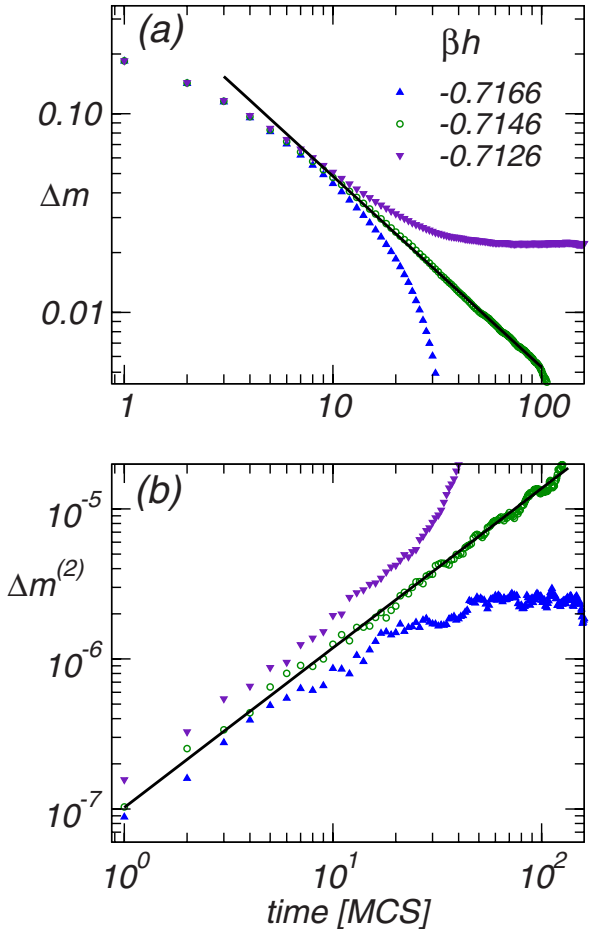

FIG. 1. Short time behavior of the first and second moments of $\Delta m$ for the Curie-Weiss-Ising model. Full (black) lines correspond to power law fits at $h=-0.7146=h_{\mathrm{sp}}^{(-)}$.

Figure 2 shows the numerical derivative obtained from runs at three values of $h$. Again these data can be fitted with a power law with exponent $w=2.02 \pm 0.02$.

Using the scaling relations [Eqs. (5) and (6)] from Sec. II and the values of $x$ and $w$, we obtain

$$
\beta=0.49 \pm 0.01
$$

and assuming $d_{c}=6$ from Eqs. (3) and (5) we have

$$
z=2.01 \pm 0.03 .
$$

The exponent $\nu$ is obtained from Eq. (6),

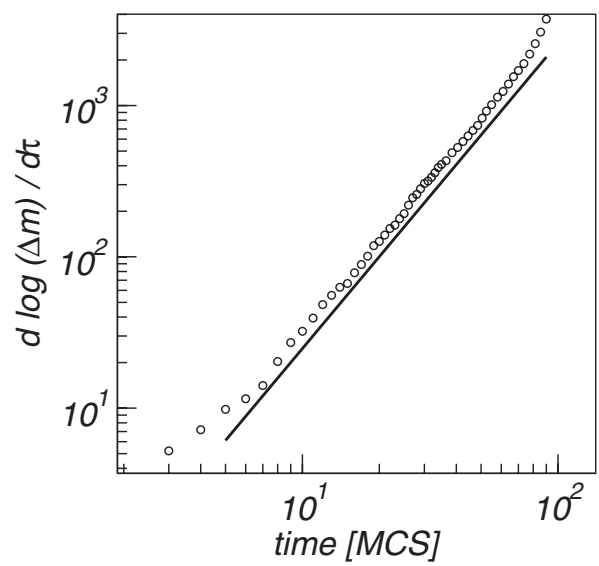

FIG. 2. Short time behavior of the derivative of $\log \Delta m$ with respect to the reduced field $\tau \equiv\left(h-h_{\mathrm{sp}}^{(-)} / h_{\mathrm{sp}}^{(-)}\right)$evaluated at $\tau=0$; the straight line corresponds to a power law with exponent $w=2.02$. 


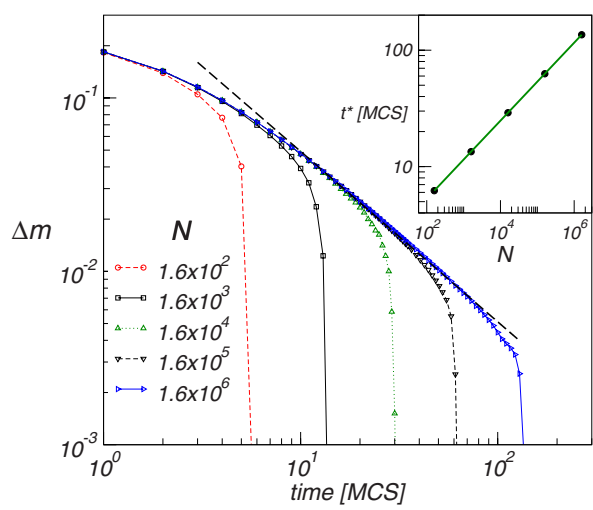

FIG. 3. Short time behavior of the order parameter $\Delta m$ at $h=h_{\mathrm{sp}}^{(-)}$for different system size $N$. The dashed (black) line corresponds to a power law fit to the $N=1.6 \times 10^{6}$ curve. Inset: $t^{*}$ defined as the time for $\Delta m$ to reach $10^{-3} \mathrm{vs}$ $N$.

$$
\nu=0.249 \pm 0.004
$$

Thus, in this test case we find that the spinodal field as well as the (static) critical exponents determined through STD are in excellent agreement with the theoretical results (17), (24), and (25).

We have also explored finite size effects which we use to obtain another determination of $z$. Figure 3 shows the evolution of $\Delta m$ for systems with number of spins ranging from $N=1.6 \times 10^{2}$ to $N=1.6 \times 10^{6}$ at $h=h_{\mathrm{sp}}^{(-)}$. The same power law fit of Fig. 1(a) is also included here. There are clear finite size effects and it is seen that the power-law fit is valid for $t>t_{\text {mic }} \sim 10$ up to a time which increases with system size. The time $t_{\text {mic }}$ is a microscopic time scale and it is the time required to sweep away the microscopic short-wave behavior. ${ }^{18}$ We define a time scale $t^{*}$, so that for $t>t^{*} \Delta m$ has abandoned the power law and is rapidly evolving toward its equilibrium value (here we take $t^{*}$ as the time when $\Delta m=3$ $\times 10^{-3}$ ). Figure 3 (inset) shows $t^{*}$ versus $N$ in a log-log scale. Such behavior can be understood by considering the evolution of the correlation length $\xi$. In the critical regime, it increases following the relation

$$
\xi \propto t^{1 / z}
$$

We expect deviations from this critical law when the correlation length becomes of the order of the linear dimension of the system $\xi \sim L$. If we assume that our system of $N$ spins behaves like one with linear dimension $L=N^{1 / d_{c}}$, the deviation would appear when $\xi \sim N^{1 / d_{c}}$ or $\left(t^{*}\right)^{1 / z} \propto N^{1 / d_{c}}$, and therefore

$$
t^{*} \propto N^{u}, \quad u=\frac{z}{d_{c}} .
$$

In this way we can use finite size effects to extract $z$. From the fit of Fig. 3 (inset) we get $u=0.34 \pm 0.01$, which together with $d_{c}=6$ gives $z=2.06 \pm 0.06$, in excellent agreement with the previous estimate.

We conclude from this test that the STD technique identifies the (thermodynamic) spinodal points consistently with the static results. We proceed now to a short-range model, where static approaches are unsuitable.

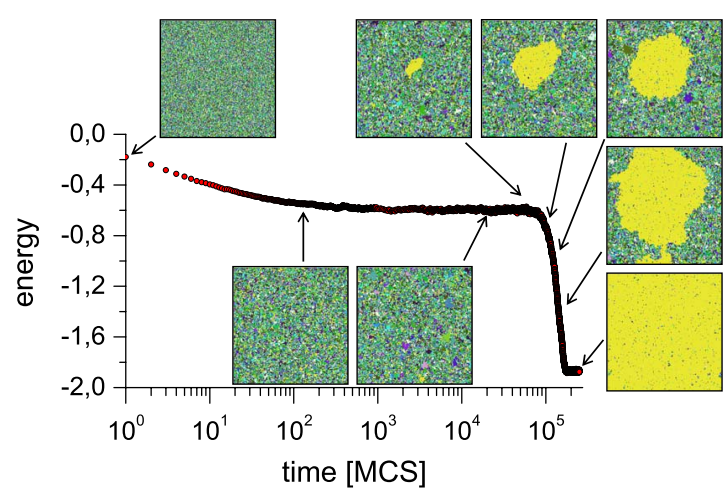

FIG. 4. A typical single-sample energy per spin vs time plot after a quench from infinite temperature to $T=0.99 T_{t}$ for $q=24$ and $L=200$. Snapshots at selected times are also shown with color coding for spin values.

\section{SPINODAL POINTS IN THE SHORT-RANGE POTTS MODEL}

We consider now the nearest-neighbor $q$-state Potts model $^{14}$ on the square lattice with Hamiltonian

$$
\mathcal{H}_{P}=-J \sum_{\langle i, j\rangle} \delta\left(\sigma_{i}, \sigma_{j}\right), \quad J>0,
$$

where the sum runs now over all the pairs of nearestneighbor sites. The two-dimensional Potts model undergoes a second order phase transition for $q=2,3,4$ and a first order one for $q>4$. In the square lattice, the transition temperature is known exactly to be ${ }^{22} T_{t}(q) / J=1 / \ln (1+\sqrt{q})$. For $q$ larger but near $q=4$, however, the transition is very weak. As Binder $^{23}$ pointed out, the pseudospinodal temperatures $T_{\mathrm{sp}}^{(+)}$ and $T_{\mathrm{sp}}^{(-)}$are extremely close to $T_{t}\left(\left[T_{t}-T_{\mathrm{sp}}^{(-)}\right] / T_{t} \lesssim 10^{-3}\right.$ for $q=5,6)$. It is thus very hard to establish whether the pseudospinodals are different from the transition temperature in the thermodynamic limit (Binder ${ }^{23}$ concluded that systems at least as large as $1000 \times 1000$ would be required), and the question of the existence of metastable phases in the thermodynamic limit has remained controversial. ${ }^{11,24,25}$

We have determined the lower spinodal of this model using the short time dynamics as well as the pseudospinodal from metaequilibrium measurements. We have done Monte Carlo simulations with single-spin-flip Metropolis dynamics on square lattices with $N=L \times L$ sites ( $L$ ranging between $L=200$ and $L=4000$ ) and periodic boundary conditions for $q=9,12,24,48,96$, and 192. The runs were quenches from infinite temperature, i.e., at constant $T$ [typically below $T_{t}(q)$ ] but starting from a random configuration.

\section{A. Pseudospinodal}

For a narrow range of quench temperatures $T<T_{t}$ the system gets stuck in a high energy paramagnetic metastable state, where it remains for some random time, after which it relaxes (relatively quickly) to the equilibrium ferromagnetic state through a nucleation process. A typical energy versus time curve is shown in Fig. 4, together with some snapshots of spin configurations, illustrating the nucleation process. The time for the formation of a critical nucleus has a lognormal distribution, whose average $\tau_{\text {nuc }}$ depends both on ${ }^{26} T$ and $L$. To find the pseudospinodal, we look for a divergence 


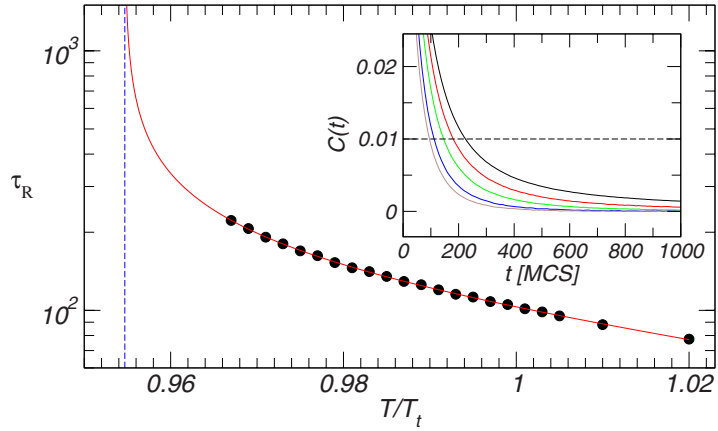

FIG. 5. Relaxation time vs $T / T_{t}$ for $L=1000$ and $q=96$. The continuous line is a fit to the data up to $T / T_{t}<1.02$ using Eq. (33). Error bars are smaller than the symbol size. The inset shows the corresponding stationary correlation function vs $t$ for temperatures ranging from $T=1.005 T_{t}$ (left) to $T$ $=0.967 T_{t}$ (right). The vertical dashed line is $T=T^{*}$.

of the relaxation time of the metaequilibrium phase, so we must ensure that the measurements are done at times shorter than $\tau_{\text {nuc }}(T)$ in order to avoid entering the regime where domains of the stable phase have begun to grow. For this we consider the two-time autocorrelation function

$$
C\left(t_{1}, t_{2}\right)=\frac{q}{q-1}\left\langle\frac{1}{N} \sum_{i}^{N} \delta\left(\sigma_{i}\left(t_{1}\right), \sigma_{i}\left(t_{2}\right)\right)-\frac{1}{q}\right\rangle,
$$

where $t_{1}$ is the time elapsed since the quench $t_{2}>t_{1}$ and the average is taken over different realizations of the thermal noise. Out of equilibrium, $C\left(t_{1}, t_{2}\right)$ depends on both $t_{1}$ and $t_{2}$ while in a stationary (meta)stable state it depends only on the time difference $t \equiv t_{2}-t_{1}$. To compute the relaxation time $\tau_{R}$, we use correlation data only from the regime where it is independent of $t_{1}$, thus staying at temperatures above the metastability limit. The typical behavior of $C(t)$ in this regime is shown in the inset of Fig. 5 for $q=96$. It is clear that the relaxation time is growing as one goes deeper into the metastable region. We defined $\tau_{R}$ as the time at which $C(t)$ falls below some threshold $C_{\text {thr }}$ (see inset of Fig. 5). Varying $C_{\text {thr }}$ within a reasonable range we obtained similar results; we have included this arbitrariness in the error estimates. The behavior of $\tau_{R}$ as a function of temperature is plotted in Fig. 5 for $q=96$.

From these data we estimated the pseudospinodal temperature $T^{*}$. This is not an easy task: Far from the divergence, corrections to power-law scaling can be important, but on the other hand the pseudospinodal cannot be approached with equilibrium measurements. The estimates of $T^{*}$ and associated exponents will necessary have relatively large uncertainties. To proceed, we fit the relaxation time with a scaling form ${ }^{27}$

$$
\tau_{R}=A\left(\frac{T-T^{*}}{T_{t}}\right)^{-b}\left[1+B\left(\frac{T-T^{*}}{T_{t}}\right)^{c}\right],
$$

fixing $c$ to different values. The best fit according to the correlation coefficient $\left(R^{2}=0.99989\right)$ was obtained for $c=1$, for which the scaling correction to the power law is around $5 \%$. However other values also give good fits. We find that while the estimates of $b$ depend on the form of the chosen correction, the estimate of $T^{*}$ is within the interval $T^{*}$ $=0.95 \pm 0.01$. We cannot give an accurate estimate of $b$ other
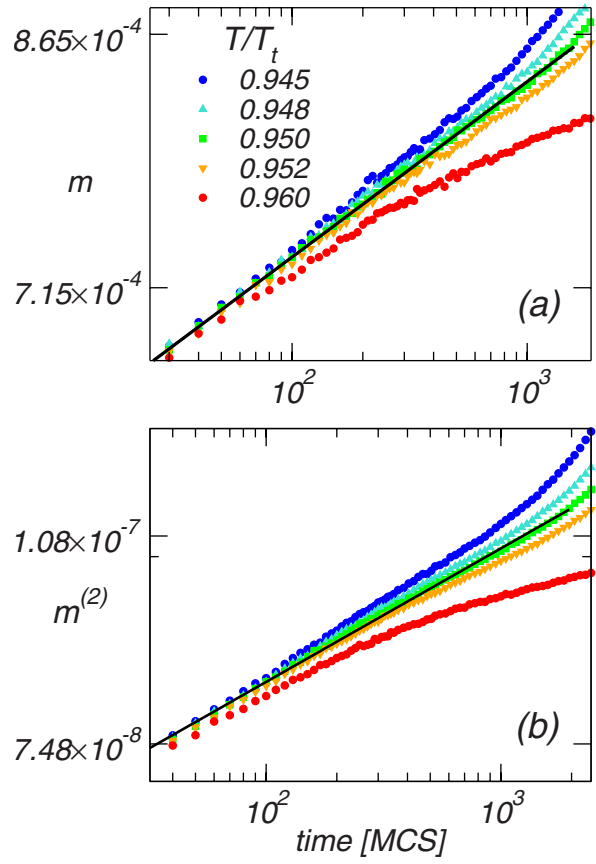

FIG. 6. Short time behavior in a supercooled state at different temperatures for $q=96$ and $L=480$. Full lines are power law fittings for $T=0.950 T_{t}$. (a) Order parameter $m$. (b) Second moment $m^{(2)}$.

than stating that the divergence is weak with $b$ ranging from 0.2 to 0.85 . This is enough for our purpose of comparing $T^{*}$ with the spinodal obtained from STD, which we compute in Sec. IV B.

\section{B. The spinodal through STD}

We now attempt to find the spinodal using the STD. We consider the dynamic behavior of the magnetization [Eq. (23)] and the corresponding second moment, ${ }^{18}$

$$
m^{(2)}=\frac{q}{(q-1)^{2}} \sum_{j=1}^{q}\left\langle\left(\frac{1}{N} \sum_{i=1}^{N} \delta\left(\sigma_{i}, \sigma_{j}\right)-\frac{1}{q}\right)^{2}\right\rangle,
$$

starting from a completely disordered state. The results for $q=96$ are shown in Fig. 6. We observe a clear power law increase, $m \sim t^{\theta}$ and $m^{(2)} \sim t^{\omega}$, spanning two decades in time for $T=T_{\mathrm{sp}}^{(-)}=(0.950 \pm 0.002) T_{t}$. The exponents we find are rather small $(\theta \approx 0.06$ and $\omega \approx 0.1)$.

At variance with the mean-field case, the STD results show no noticeable finite size effects. Figure 7 shows $m^{(2)} L^{2}$

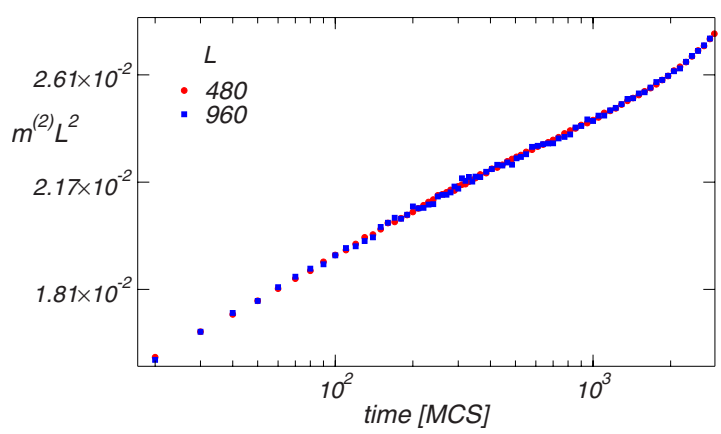

FIG. 7. Short time behavior of the second moment $m^{(2)}$ at $T=0.95 T_{t}$ for different system sizes $(L)$ and $q=96$ Potts model. 


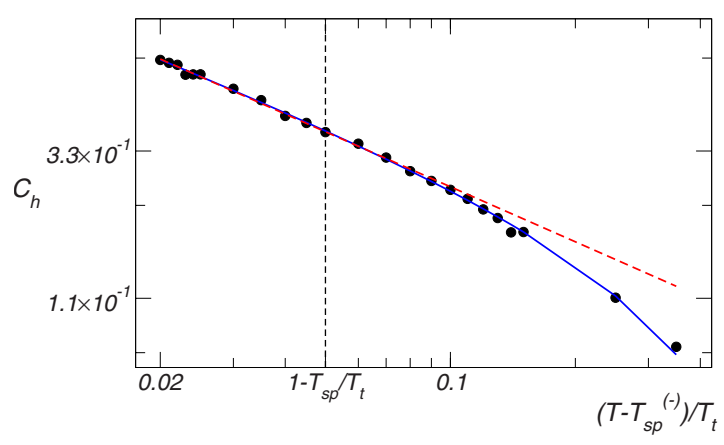

FIG. 8. Specific heat as a function of $T-T_{\mathrm{sp}}^{(-)}$in the metastable state for $q$ $=96$ and $L=480$. The full (blue) line is a fit with a law $A\left(T-T_{\mathrm{sp}}^{(-)}\right)^{\alpha}[1$ $\left.+B\left(T-T_{\mathrm{sp}}^{(-)}\right)\right]$. The dashed (red) line is a power law fit using the data for $\left(T-T_{\mathrm{sp}}^{(-)}\right) / T_{t}<0.15$. Error bars are smaller than the symbol size. The vertical dashed line is $T=T_{t}$.

versus time at the spinodal temperature $T_{\mathrm{sp}}^{(-)}=0.950 T_{t}$ for $L$ $=480$ and 960. The curves are almost indistinguishable, indicating that the spinodal critical regime lasts for a sizeindependent time. Clearly, at this time correlations have not yet reached lengths of the order of the smallest system size. This is in contrast to the mean-field case (Fig. 3), where it seems that the number of spins that must become correlated before faster growth of the stable phase begins grows with $N$. Again, we see that the span of the power law is limited, so that the values of the exponents are not very accurate. However, our interest is to establish the existence of a powerlawlike regime to define $T_{\mathrm{sp}}$ and to compare its value to the pseudospinodal.

The temperature $T_{\mathrm{sp}}^{(-)}$identified by STD is equal to the pseudospinodal (here defined as the apparent divergence of the relaxation time). The near instability of the system at $T_{\mathrm{sp}}^{(-)}$ also shows up in the specific heat $C_{h}$ and magnetic susceptibility $\chi_{T}$. We computed these quantities in the metastable regime, where we find the same values either through fluctuations of the energy (magnetization) or by a numerical derivative with respect to temperature (field). Plots for $q=96$ are shown in Figs. 8 and 9. $C_{h}$ and $\chi_{T}$ grow in a way compatible with a divergence at $T=T_{\mathrm{sp}}$. However, the available equilibrium data span too short a range to get a reliable estimate of the critical exponents.

Let us remark that Fig. 6 is not completely equivalent to

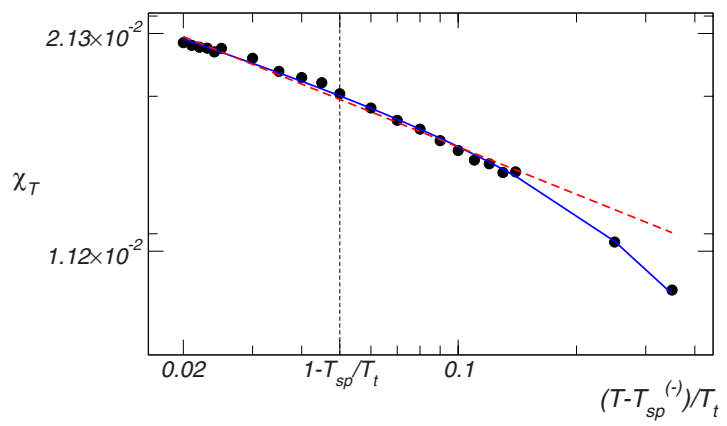

FIG. 9. Magnetic susceptibility as a function of $T-T_{\mathrm{sp}}^{(-)}$in the metastable state for $q=96$ and $L=480$. The full (blue) line is a fit with a law $A(T$ $\left.-T_{\mathrm{sp}}^{(-)}\right)^{\alpha}\left[1+B\left(T-T_{\mathrm{sp}}^{(-)}\right)\right]$. The dashed (red) line is a power law fit using the data for $\left(T-T_{\mathrm{sp}}^{(-)}\right) / T_{t}<0.15$. Error bars are smaller than the symbol size. The vertical dashed line is $T=T_{t}$.
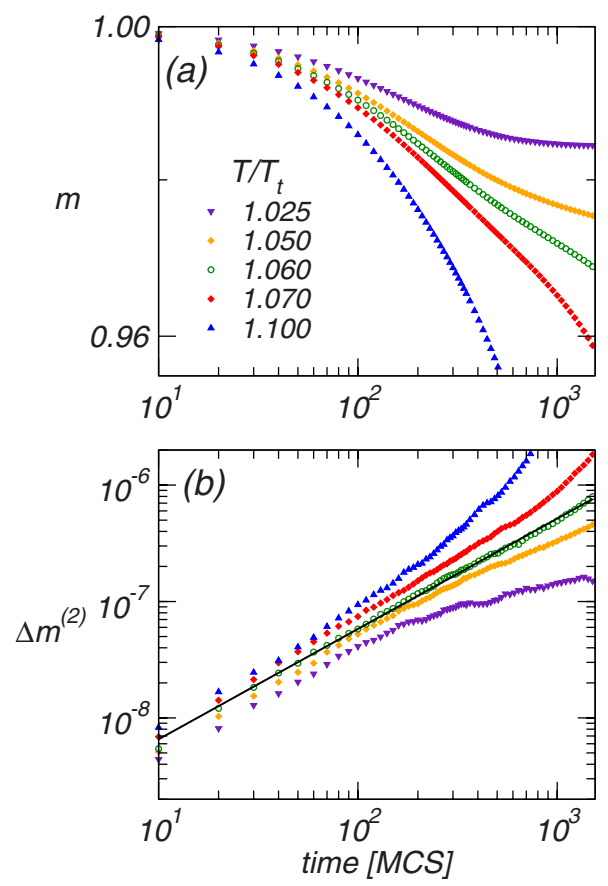

FIG. 10. Short time behavior in a superheated state at different temperatures for $q=96$ and $L=480$. Full line in (b) is a power-law fit for $T=1.060 T_{t}$. (a) Order parameter $m$. (b) Second moment $m^{(2)}$.

Fig. 1 (Curie-Weiss-Ising). The second moment of the order parameter [part (b) of both figures] behaves the same in both cases but not the order parameter itself. This is because of the initial conditions that must be used. In general, at a critical point, if one starts with a large value of $\Delta m$, one observes a power-law decay toward zero (the Ising spinodal corresponds to this case). If one starts from near zero $\Delta m$, the order parameter is first observed to increase with a power law, then to decay again to zero. ${ }^{12}$ This initial increase is governed by an exponent $\theta$, which cannot be determined from the equilibrium critical exponents alone [see Eq. (2).$^{12,18}$ The latter is the situation in the Potts model at $T$ $=T_{\mathrm{sp}}^{(-)}$. In an equilibrium critical point, one is free to choose the initial condition, so both situations can be observed. In the Potts $T_{\mathrm{sp}}^{(-)}$case, however, we cannot start with a high value of $m$ because that would place us automatically outside the metastable phase we are trying to observe, so we can only hope to see the initial increase (the corresponding decrease at longer times is masked by the evolution to the stable phase). In the Curie-Weiss-Ising case, we could in principle set $\Delta m \approx 0$ and see something similar to Fig. 6(a). We have failed to observe such increase, however, indicating that the exponent $\theta$ is zero. Indeed, in equilibrium it is known that $\theta=0$ in the mean-field case. ${ }^{12}$

On the other hand, in the upper spinodal point of the Potts model, $T=T_{\mathrm{sp}}^{(+)}>T_{t}$, we can start with an ordered initial state, and we should again observe $m$ decrease. Indeed, we have simulated the short time dynamics for $q=96$ and $N$ $=480$ taking an ordered initial state and tuning the temperature above the transition value $T_{t}$. Figure 10 shows the magnetization and its second moment measured in the same way as in Fig. 1 for different temperatures. In Fig. 10(b) we can identify a nice power law in the second moment for 


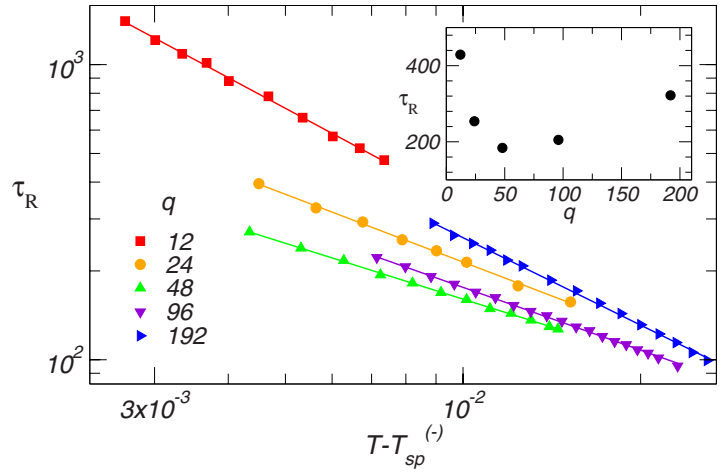

FIG. 11. Relaxation time as a function of $T-T_{\mathrm{sp}}^{(-)}$for $L=1000$ and different values of $q$. Error bars are smaller than the symbol sizes. Note, however, that due to the error in the determination of $T_{\mathrm{sp}}$, the points could be uniformly shifted horizontally. The continuous lines are power law fits. Inset: $\tau_{R}$ as a function of $q$ for a fixed temperature $T-T_{\mathrm{sp}}=0.008$.

$T_{\mathrm{sp}}^{(+)} / T_{r}=1.06 \pm 0.01$ over more than two decades. The fitted exponent is $y \approx 0.90$. At this temperature, Fig. 10(a) shows that the magnetization decreases in the same way as in Fig. 1(a). The main difference is that the spinodal magnetization in the latter case is known exactly, while in the Potts case it is unknown and cannot be estimated with good precision with these data. Thus we do not see a power law in the $m$ plot, and we rely on $m^{(2)}$ to find the spinodal.

Finally, we have repeated the above simulations for several values of $q$, computing the relaxation time and the STD behavior. In all cases we found that the spinodal $T_{\mathrm{sp}}^{(-)}$found by STD is compatible with the pseudospinodal $T^{*}$ from metaequilibrium measurements. The relaxation time as a function of temperature for several $q$ and $L=1000$ is given in Fig. 11 (the same results were found for $L=2000$ and 4000). This calculation was done using the same threshold $C_{\mathrm{thr}}=0.01$ for all values of $q$. We observed that for fixed $T-T_{\text {sp }}$, the relaxation time is nonmonotonic as a function of $q$ with a minimum around $q \approx 50$ (see inset of Fig. 11), and the same nonmonotonic behavior is observed in the exponents. We do not have an explanation for this behavior. However, the growth of the relaxation time for large values of $q$ is consistent with the appearance of a true singularity, since the $q \rightarrow \infty$ limit is mean field. ${ }^{28}$ We also observed that the kinetic spinodal is strongly $q$-dependent and therefore the minimum distance to $T_{\mathrm{sp}}^{(-)}$available for metaequilibrium measurements increases with $q$.

In Fig. 12 we show $\left(T_{t}-T_{\mathrm{sp}}^{(-)}\right) / T_{t}$ versus $q$ (we have included the "pseudocritical" temperatures found in Ref. 15 for $q=5,7)$. We see that $T_{\mathrm{sp}}^{(-)}$systematically departs from $T_{t}$ as $q$ increases. In fact, the data are very well fitted by the logarithmic form $A \log ^{a}(1+q-4)$ with $a=2.81$, in qualitative agreement with the behavior of the mean field or CurieWeiss-Potts, and the Bethe lattice solution with coordination number ${ }^{29,30} z=3$, as expected in the large $q$ limit. $^{28}$

\section{CONCLUSIONS}

We have shown that it is possible to define the spinodal point through the short time dynamic behavior. The STD can be used to detect a point in the phase diagram where the dynamics is critical (albeit for a finite time). In mean-field

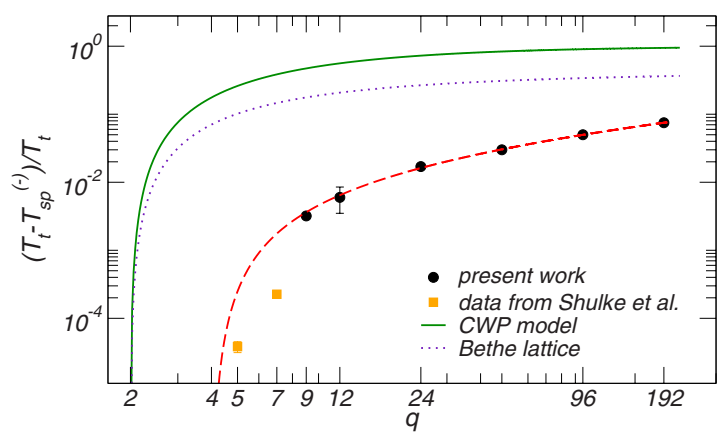

FIG. 12. $\left(T_{t}-T_{\mathrm{sp}}^{(-)}\right) / T_{t}$ vs $q$ compared to mean-field predictions. Data for $q$ $=5,7$ are from Ref. 15. The dashed (red) line is a fit to $A \log ^{a}(1+q-4)$ with $A=0.0007$ and $a=2.81$.

systems this coincides with the thermodynamic spinodal defined through the vanishing of second derivatives of the free energy, while in finite-dimensional systems it serves as a definition of spinodal, a point where (meta)equilibrium measurements are impossible (since it is beyond the metastability limit or kinetic spinodal). In the two-dimensional Potts model, we found that the spinodal defined in this way coincides with the pseudospinodal found through fitting and extrapolation of metaequilibrium relaxation times. Our results are consistent with the scaling behavior associated with a growing correlation length. In particular, (pseudo)critical exponents can be measured using STD, which in the mean-field case we have checked with the analytical result.

For the Potts model, this method gives a spinodal temperature different from the transition temperature at all $q$ where the transition is first order even in the thermodynamic limit. Since the spinodal provides only a bound for the metastability limit, this does not settle the question of the existence of a metastable phase, but it does show that the apparent convergence of spinodal and transition temperatures is due to the extremely weak nature of the transition for low values of $q$.

Apart from the conceptual advantage of allowing a definition of spinodal points avoiding equilibration issues, the STD method may prove to be practically useful in establishing bounds for metastability limits. This would be especially welcome, for instance, in systems with very slow dynamics such as glass forming liquids, where metaequilibrium measurements are out of the question (a direction in which some work is in progress), and even more so if this technique could be implemented experimentally.

Finally, let us remark that although our results show that one can define a spinodal (which we call thermodynamic to distinguish it from the kinetic spinodal) through a criticallike dynamics, this behavior is transient (except in mean field), so that we do not conclude that any thermodynamic potential is singular at this point. The thermodynamic spinodal in this sense is better understood as similar to the pseudospinodal but defined in a way free from the equilibration and extrapolation issues that pervade the pseudospinodal. In the two-dimensional Ising model, the pseudospinodal has been shown to be related to zeros of the partition function at complex values of temperature and field, ${ }^{31}$ which approach the real plane as the range of the interaction is increased. We 
may conjecture a similar scenario for the Potts model, whose thermodynamical behavior can be determined by zeros of its partition function in the complex temperature plane, ${ }^{32}$ and where evidence has been found of singular behavior (with divergent thermodynamical quantities) associated with some of those zeros ${ }^{33}$ for $q>4$. It is tempting to conjecture that the (pseudo)critical dynamics observed here is associated with such zeros, and that the longer its lifetime the nearer these zeros are to the real plane. This remains to be studied, however, but it is an interesting issue to investigate especially in short-range interacting systems, where a pseudosingularity is observed even if the mechanism of a vanishing free energy barrier for long wavelength fluctuations is in principle excluded.

\section{ACKNOWLEDGMENTS}

We thank Andrea Cavagna, Irene Giardina, and Víctor Martín-Mayor for many discussions on the issue of metastability and spinodals and M. Ibáñez de Berganza for useful discussions about the Potts model. This work was supported by CONICET, Universidad Nacional de Córdoba, Universidad Nacional de La Plata, ANPCyT grants (Grant Nos. PICT 20472/04 and PICT 33305/05) (Argentina), and ICTP (Italy).

${ }^{1}$ J. J. Binney, N. J. Dowrick, A. J. Fisher, and M. E. J. Newman, The Theory of Critical Phenomena (Oxford University Press, New York, 1992).

${ }^{2}$ K. Binder, Rep. Prog. Phys. 50, 783 (1987).

${ }^{3}$ K. Binder, Philos. Mag. Lett. 87, 799 (2007).

${ }^{4}$ W. Kauzmann, Chem. Rev. (Washington, D.C.) 43, 219 (1948).

${ }^{5}$ A. Z. Patashinskii and B. I. Shumilo, Sov. Phys. JETP 50, 712 (1979).
${ }^{6}$ A. Z. Patashinskii and B. I. Shumilo, Sov. Phys. Solid State 22, 655 (1980).

${ }^{7}$ S. B. Kiselev and J. F. Ely, Physica A 299, 357 (2001).

${ }^{8}$ H. Larralde, F. Leyvraz, and D. P. Sanders, J. Stat. Mech.: Theory Exp. 2006, P08013 (2006)

${ }^{9}$ P. M. Chaikin and T. C. Lubensky, Principles of Condensed Matter Physics (Cambridge University Press, Cambridge, England, 2000).

${ }^{10}$ D. W. Herrmann, W. Klein, and D. Stauffer, Phys. Rev. Lett. 49, 1262 (1982).

${ }^{11}$ L. A. Fernández, J. J. Ruiz-Lorenzo, M. P. Lombardo, and A. Tarancón, Phys. Lett. B 277, 485 (1992).

${ }^{12}$ H. K. Janssen, B. Schaub, and B. Schmittmann, Z. Phys. B: Condens. Matter 73, 539 (1989).

${ }^{13}$ B. Zheng, in Computer Simulation Studies in Condensed-Matter Physics, edited by D. P. Landau, S. P. Lewis, and H.-B. Schüttler (Springer, New York, 2006).

${ }^{14}$ F. Y. Wu, Rev. Mod. Phys. 54, 235 (1982).

${ }^{15}$ L. Schülke and B. Zheng, Phys. Rev. E 62, $7482(2000)$.

${ }^{16}$ R. A. Monetti and E. V. Albano, Europhys. Lett. 56, 400 (2001).

${ }^{17}$ G. P. Saracco and E. V. Albano, J. Chem. Phys. 118, 4157 (2003).

${ }^{18}$ B. Zheng, Int. J. Mod. Phys. B 12, 1419 (1998).

${ }^{19}$ J. D. Gunton and M. C. Yalabik, Phys. Rev. B 18, 6199 (1978).

${ }^{20}$ L. Mittag and M. J. Stephen, J. Phys. A 7, L109 (1974).

${ }^{21}$ W. Klein, Phys. Rev. Lett. 47, 1569 (1981).

${ }^{22}$ T. Kihara, Y. Midzuno, and T. Shizume, J. Phys. Soc. Jpn. 9, 681 (1954).

${ }^{23}$ K. Binder, J. Stat. Phys. 24, 69 (1981).

${ }^{24}$ J. L. Meunier and A. Morel, Eur. Phys. J. B 13, 341 (2000).

${ }^{25}$ M. Ibáñez de Berganza, V. Loreto, and A. Petri, e-print arXiv:cond-mat/ 0706.3534.

${ }^{26}$ E. E. Ferrero and S. A. Cannas, Phys. Rev. E 76, 031108 (2007).

${ }^{27}$ N. Goldenfeld, Lectures on Phase Transitions and the Renormalization Group (Addison-Wesley, Reading, MA, 1992).

${ }^{28}$ P. Pearce and R. Griffiths, J. Phys. A 13, 2143 (1980).

${ }^{29}$ F. Peruggi, F. di Liberto, and G. Monroy, J. Phys. A 16, 811 (1983).

${ }^{30}$ F. Wagner, D. Gresing, and J. Heide, J. Phys. A 33, 929 (2000).

${ }^{31}$ N. Gulbahce, H. Gould, and W. Klein, Phys. Rev. E 69, 036119 (2004).

${ }^{32}$ R. Kenna, J. Phys. A 31, 9419 (1998).

${ }^{33}$ V. Matveev and R. Shrock, Phys. Rev. E 54, 6174 (1996). 